\title{
MATRIX VERSIONS OF YOUNG'S INEQUALITY
}

\author{
TOMAŽ Kosem
}

Abstract. A matrix majorization version of the general Young's inequality $x y \leqslant \Phi(x)+\Psi(y)$ is presented. Multivariate Young's inequality is extended to the matrix setting by means of the geometric mean of positive semidefinite matrices. Also some refined Hilbert-Schmidt norm generalizations of Young's inequality are given and a symmetrized Young's inequality for unitarily invariant norms is proved.

Mathematics subject classification (2000): 15A45, 15A60.

Keywords and phrases: unitarily invariant norm, Young's inequality, Young's function, positive-semidefinite matrix, eigenvalue, majorization, convex function, geometric mean, Hilbert-Schmidt norm, Hadamard product.

\section{REFERENCES}

[1] T. ANDO, Matrix Young inequalities, Operator theory in function spaces and Banach lattices, 3338, Oper. Theory Adv. Appl., 75, Birkhäuser, Basel, 1995.

[2] T. ANDo, C. K. LI, R. MATHiAs, Geometric means, Linear Algebra Appl., 385 (2004), 305-334.

[3] J. S. Aujla, F. C. Silva, Weak majorization inequalities and convex functions, Linear Algebra Appl., 369 (2003), 217-233.

[4] E. F. Beckenbach, R. Bellman, Inequalities, Second revised printing, Ergebnisse der Mathematik und ihrer Grenzgebiete. Neue Folge, Band 30, Springer-Verlag, New York, Inc., 1965.

[5] R. Bhatia, Matrix Analysis, GTM 169, Springer-Verlag, New York, 1997.

[6] R. Bhatia, J. Holbrook, Riemannian geometry and matrix geometric means, Linear Algebra Appl., 413 (2006), 594-618.

[7] R. Bhatia, F. Kittaneh, Notes on matrix arithmetic-geometric mean inequalities, Linear Algebra Appl., 308 (2000), 203-211.

[8] R. Cooper, Notes on certain inequalities (1), J. London Math. Soc., 2 (1927), 17-21.

[9] T. FURUTA, M. YANAGIDA, Generalized means and convexity of inversion for positive operators, Amer. Math. Monthly, 105 (1998), 258-259.

[10] O. Hirzallah, F. KitTaneh, Matrix Young inequalities for the Hilbert-Schmidt norm, Linear Algebra Appl., 308 (2000), 77-84.

[11] H. KosAKI, Arithmetic-geometric mean and related inequalities for operators, J. Funct. Anal., 156, 2 (1998), 429-451.

[12] R. MATHIAS, An arithmetic-geometric-harmonic mean inequality involving Hadamard products, Linear Algebra Appl., 184 (1993), 71-78.

[13] D. S. Mitrinović, Analytic inequalities, Die Grundlehren der mathematischen Wissenschaften, Band 1965, Springer-Verlag, New York-Berlin, 1970.

[14] A. Oppenheim, Note on Mr. Cooper's Generalization of Young's Inequality, J. London Math. Soc., 2 (1927), 21-23.

[15] M. M. RaO, Z. D. Ren, Theory of Orlicz spaces, Monographs and Textbooks in Pure and Applied Mathematics, 146, Marcel Dekker, Inc., New York, 1991.

[16] D. RÜthing, On Young's inequality, Internat. J. Math. Ed. Sci. Tech., 25, 2 (1994), 161-164.

[17] X. Zhan, Matrix inequalities, LNM 1790, Springer-Verlag, Berlin, 2002. 